\title{
An ISAR-SAR based Method for Indoor Localization using Passive UHF RFID System with Mobile Robotic Platform
}

\author{
Zheng Liu, Zhe Fu, Tongyun Li, Ian H. White, Richard V. Penty, and Michael Crisp
}

\begin{abstract}
A novel RFID inverse synthetic aperture radar (ISAR)-synthetic aperture radar (SAR) localization method is proposed and demonstrated which exploits reference tags with known locations to estimate the trajectory of a mobile platform and locate target tags with unknown locations. A system that integrates a mobile robot with an integrated RFID reader and antenna is used to obtain phase measurements of both reference and target tags when the robot is moving along its trajectory. The ISAR algorithm estimates the antenna trajectory and a novel ISAR-SAR loop then adjusts the estimated trajectory. The estimated trajectory is combined with phase measurements of the target tags to determine their location. The performance is compared to SAR using a LiDAR measured antenna trajectory. Similar localization accuracy (15 cm MAE) is achieved using LiDAR-SAR or ISAR-SAR method with a straight-line trajectory and higher accuracy $(6 \mathrm{~cm})$ is achieved using ISAR-SAR method than LiDAR-SAR method $(8 \mathrm{~cm})$ with an L-shape trajectory.
\end{abstract}

Keywords-RFID; SAR; ISAR; Localization; reference tags

\section{INTRODUCTION}

Over the past decade, passive Ultra High Frequency-Radio Frequency Identification (UHF-RFID) technology has attracted increasing interest due to its advantages such as contactless communication, multi-object recognition, and low-cost [1, 2] and has become widely used in various scenarios such as inventory interrogation in warehouses, factories and stores [2, 3]. Recently, there is an increasing requirement for accurate positional information of materials and products in an indoor environment where the Global Positioning System (GPS) is unable to reach and is too expensive $[4,5]$. By integrating passive RFID technology with a mobile robotic platform, it could provide high localization accuracy at a low cost [6-8].

Various methods have been proposed for indoor localization using passive RFID tags, e.g. phase-based, received signal strength indicator (RSSI)-based, angle of arrival (AoA)-based, time of arrival (ToA)-based real-time localization systems (RTLS) [9]. Among these methods, phase-based localization is more robust and stable in complex indoor environments. There are also many different phase-based methods to locate target objects [10-23]. Synthetic Aperture Radar (SAR), which exploits the mobility of a mobile platform, is a promising and

Zheng Liu, Zhe Fu, Tongyun Li, Richard V. Penty, and Michael Crisp are with the Electrical Division, Department of Engineering, University of Cambridge, Cambridge, CB3 1PZ, U.K. (e-mail: zl390@cam.ac.uk).

Ian H. White is with the, University of Bath, Claverton Down, Bath BA2 7AY, U.K. popular algorithm to estimate the location of targets with minimal radio hardware $[11,12]$.

The mobile platform to which the reader and the antenna are attached could be a robotic arm [15], an unmanned ground vehicle (UGV) $[10-13,23,24]$ or an unmanned aerial vehicle (UAV) $[16,17,22]$. The trajectory of the moving antenna is required to perform SAR and locate target tags. To estimate the antenna trajectory, previous studies have exploit optical systems including motion capture system $[11,12]$, cameras and light detection and ranging (LiDAR) based hybrid system [13, 23-25], and GPS [16, 17, 22]. DiGiampaolo and Martinelli proposed a tracking algorithm, which is based on Kalman filtering and exploiting the SAR method, using tags with known locations on the ceiling to estimate the trajectory of a robot [26, 27]. The SAR algorithm for outdoor RFID localization based on GPS is demonstrated in [16] which achieves a tag localization error of $2.3 \mathrm{~cm}$ along the $\mathrm{x}$-axis and $-4.4 \mathrm{~cm}$ along the $y$-axis. In [24], the phase relock method proposed another localization method focusing on unwrapping and reconstructing the phase measurements which achieves a mean error of around $17 \mathrm{~cm}$ in $\mathrm{x}$ and $\mathrm{y}$ planes using a combination of sensors to perform simultaneous localization and mapping (SLAM). A phase fingerprinting localization method is proposed in [25] of which the mean error is $15-22 \mathrm{~cm}$ and the trajectory is also estimated by SLAM with various sensors. Most localization solutions require complicated infrastructure and sensors including LiDAR to track the mobile platform. Although the LiDAR sensor is able to estimate the relative position of the robot from a reference plane using the line-of-sight (LOS) and time-of-flight (TOF) operations, the localization accuracy is limited by several environmental factors, e.g. obstructions, lighting and discontinuity of the reference plane. High performance LIDAR systems required for high measurement accuracy are also expensive. Thus, alternative or complementary solutions are required to fulfil the aim of an efficient robotic trajectory estimation in a complicated and dynamic environment.

In this paper, which is an extended version of the conference paper [28], a novel low-cost ISAR-SAR localization method is proposed and demonstrated for the first time. Passive RFID reference tags with known locations are used to find the robot trajectory. Reference tags can be placed on physically meaningful positions (e.g. shelf bays, loading areas) to allow simple registration of the coordinate system with the physical world. After estimation of the trajectory by ISAR-SAR system, unknown locations of target tags could be computed with the estimated trajectory and SAR algorithm. More details of a novel ISAR-SAR loop, which is the core part of this ISAR-SAR localization method, will be explained in the following sections. 
Since the tag locations are found relative to the location of the reference tags, the system has a degree of robustness to errors in the reference tag placement in some cases. For example, if the reference tags are on the 4 sides of a container and one wants to know if the tag is in the container or not, a relative location should give the correct answer even if the reference locations are inaccurate.

LiDAR is used in some applications for its SLAM potential to allow automated exploring of an environment while avoiding obstacles. Our approach will be of more use in structured environments where few obstacles exist, and a pre-existing map of the environment and reference locations can be provided to the robot for autonomous navigation.

In order to analyse the performance, results of ISAR-SAR based method are compared with a LiDAR-based system. Both the LiDAR measured trajectory and the estimated ISAR trajectory are used to locate target unknown tags as an evaluation of the system performance.

The remainder of the paper is organized as follows. In Section II, the RFID robotic system is introduced. The ISARSAR localization algorithm is explained in Section III which is followed by experimental setup and results in Section IV and Section V. Section IV shows results of a 1D straight-line trajectory and Section $\mathrm{V}$ shows results of a 2D L-shape trajectory. The conclusion and future work are presented in Section VI and VII respectively.

\section{SYSTEM DESIGN AND IMPLEMENTATION}

Figure 2.1 shows the full structure of the RFID robot platform. It mainly consists of an Impinj Speedway RFID reader [29] with a linearly polarised antenna mounted on a wooden pole attached to the robot, a Turtlebot3 Waffle Pi robot [30], and two Raspberry Pi controller boards [31]. Those devices are installed in different layers and connected by USB cables and power lines. The RFID reader and the Turtlebot3 Waffle Pi are remotely controlled, and their timestamps are synchronised by using a WLAN (See Fig. 2.2). The RFID reader interrogates the passive tags and records the EPC, phase information and timestamps offline processing. The speed of the robot is approximately 5 $\mathrm{cm} / \mathrm{s}$. LiDAR data can be simultaneously collected and timestamped to the RFID data for performance comparison studies.

For experimental purposes the processing is carried out offline in Matlab. The time required to process the data depends on the length of the trajectory and the performance of the processor. Currently, the duration of the processing of the trajectory and unknown tag locations is around three minutes for the linear trajectory and about five minutes for the L-shape trajectory described later in Section V.

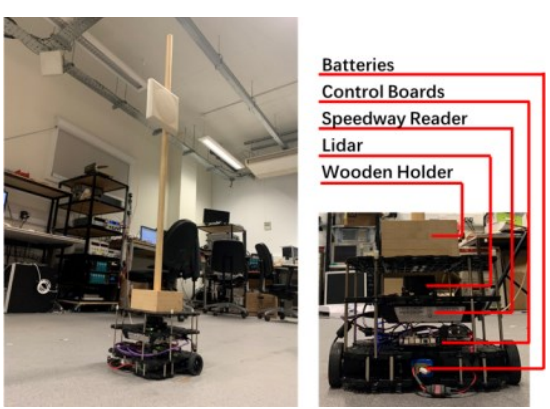

Fig. 2.1. RFID Robot [30]

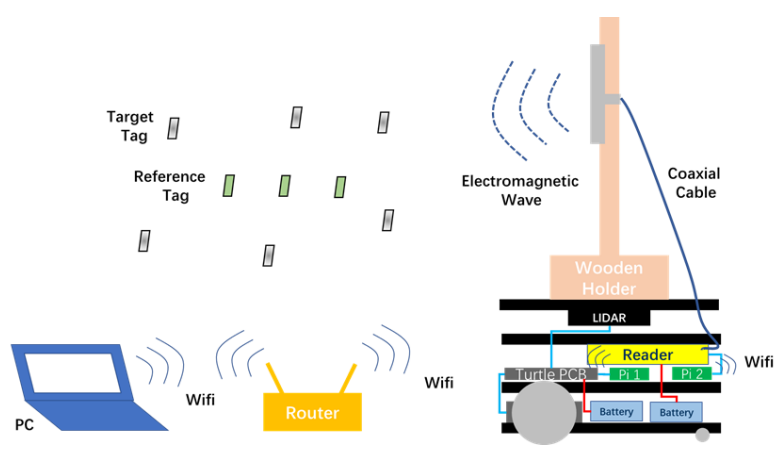

Fig. 2.2. ISAR-SAR system [30]

\section{LOCALIZATION ALGORITHM}

Fig. 3.1 shows the diagram of the main steps for ISAR-SAR processing. The ISAR algorithm is applied to measurements of the reference tag phase to estimate the best fit trajectory of the moving RFID antenna. After the estimation of the best fit trajectory, target tag locations are calculated by the SAR algorithm based on this trajectory. Since the algorithm for antenna trajectory tracking originates from the idea of SAR, the SAR method will be explained first in Section A. Section B describes the ISAR algorithm for estimating antenna trajectory. The ISAR-SAR algorithm is shown in Section C. The core part of the ISAR-SAR processing is the novel ISAR-SAR loop consisting of two main steps. Firstly, an estimated trajectory is calculated by ISAR algorithm. The second step is using the SAR algorithm to calculate the location of the reference tag based on the estimated trajectory from the previous ISAR step. The mean absolute location error between the real locations and estimated locations of reference tags is used as an index of accuracy of the trajectory. Accordingly, the loop could adjust the settings or parameters to obtain a better fitting trajectory of the moving antenna. Any unintended variation in speed and bearing will also be partially eliminated by the ISAR-SAR loop.

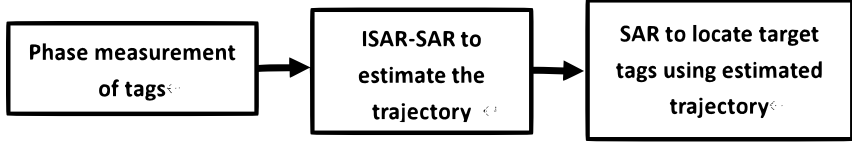

Fig. 3.1. The diagram of ISAR-SAR method 


\section{A. SAR Algorithm}

After obtaining phase values of backscattered signals from RFID tags using a mobile RFID reader with a known trajectory, a spatial ambiguity function is used to generate a probability that the tag is located at each point given the observed phase information, this can be represented as a heatmap or a pixelated holograph.

At time $t$, the location of the reader antenna is expressed as

$$
\boldsymbol{q}_{\boldsymbol{t}}=\left[x_{t}, y_{t}\right]
$$

The trajectory can be expressed as a vector of locations

$$
\mathbf{Q}=\left[q_{1}, \ldots, q_{t}, \ldots, q_{T_{1}}\right]^{T}
$$

Where $T$ is the transpose operator and each element represents a location at which information of the phase for tags is recorded.

The potential locations of the target tags can be expressed as a vector by applying a grid to the area or pixelating the potential area. The $k$-th potential position of the $n$-th tag can be written as

$$
\mathbf{b}_{n \boldsymbol{k}}^{\prime}=\left[x_{n k}^{\prime}, y_{n k}^{\prime}\right]
$$

The distance between each potential position and the location of the reader antenna can be calculated by

$$
d_{t, n k}=\left\|\boldsymbol{q}_{\boldsymbol{t}}-\mathbf{b}_{\boldsymbol{n} \boldsymbol{k}}^{\prime}\right\|
$$

Where $\|\cdot\|$ is the norm operator of the distance vector.

The expected phase for a tag at each potential location can be calculated by the following equation

$$
\phi_{t, n k}^{\prime}=\left(\phi_{0}+\frac{4 \pi d_{t, n k}}{\lambda}\right) \bmod 2 \pi
$$

Where $\phi_{0}$ represents the fixed phase shift caused by the measurement equipment such as the antenna and cable.

The relative received phase which is measured by the reader for the $n$-th target tag is

$$
\Delta \phi_{t, n}=\phi_{t, n}-\phi_{1, n}
$$

The sequence of relative received phases over time $\mathrm{T}$ could be expressed as

$$
\Delta \boldsymbol{\Phi}_{\boldsymbol{n}}=\left[0, \Delta \phi_{2, n}, \ldots, \Delta \phi_{t, n}, \ldots, \Delta \phi_{T_{1}, n}\right]^{\boldsymbol{T}}
$$

The expected relative phase of each potential tag position could also be written as

$$
\Delta \phi_{t, n k}^{\prime}=\phi_{t, n k}^{\prime}-\phi_{1, n k}^{\prime}
$$

The sequence of expected relative phases can be written as

$$
\Delta \boldsymbol{\Phi}_{n \boldsymbol{k}}^{\prime}=\left[0, \Delta \phi_{2, n k}^{\prime}, \ldots, \Delta \phi_{t, n k}^{\prime}, \ldots, \Delta \phi_{T_{1}, n k}^{\prime}\right]^{\boldsymbol{T}}
$$

The matching function is defined as

$$
\mathrm{C}_{n}=\exp \left(-j\left(\Delta \Phi_{n k}^{\prime}-\Delta \Phi_{n}\right)\right)
$$

Which is a measure of the difference between the expected and measured phase for each time step. The use of the complex field resolves the wrapping problem with the phase angles recognizing that the phase shift between $\Delta \phi$ and $2 \pi-\Delta \phi$, is $2 \Delta \phi$ rather than $2 \pi-2 \Delta \phi$.

The probability that the tag is at a particular location can be calculated as the sum of the matching function over a number of phase measurements taken at different antenna locations

$$
\mathrm{P}_{n}=\left|\sum_{t=1}^{T} \mathbf{C}_{n}\right|
$$

The final probability with multiple straight-line trajectories will be

$$
\mathrm{P}_{\text {final }}=\prod_{i=1}^{M} \mathrm{P}_{i}
$$

The estimated position of the $n$-th tag would be the location with the largest probability

$$
\boldsymbol{b}_{\boldsymbol{n}}=\underset{\mathrm{b}_{n k}^{\prime}}{\operatorname{argmax}} \mathrm{P}_{\text {final }}
$$

\section{B. ISAR Algorithm for Antenna Trajectory Tracking}

The ISAR algorithm is initially designed for the $1 \mathrm{D}$ trajectory because the robot is more stable when it moves along 1D-trajectory than along an arbitrary trajectory. As a result, when the trajectory extends to an L-shape, the turning point would lead to a larger error in direction by ISAR due to the limit of the dynamic model in the ISAR process. So, the L-shape trajectory is divided into two 1D segmentation.

The inverse-SAR (ISAR) algorithm originates from the SAR method. Instead of finding the locations of static tags at unknown locations exploiting a moving platform with a known trajectory, a number of passive RFID tags with known locations are used to estimate the moving antenna trajectory. The initial location of the antenna is assumed to be known and accurate.

At time $t-1$, the position of the reader antenna can be written as

$$
\boldsymbol{a}_{t-1}=\left[x_{t-1}, y_{t-1}\right]
$$

The antenna then moves according to the $j$-th hypothetical step

$$
v_{t, j}=[l \cos \theta, l \sin \theta]
$$

Where $l$ is the displacement of the antenna which belongs to the range $\boldsymbol{L}=\left[l_{\text {min }}, l_{\text {max }}\right]$ hence $l \in\left(l_{\text {min }}, l_{\text {max }}\right), \theta$ is the angle of direction which belongs to the range $\boldsymbol{\vartheta}=\left[\theta_{\text {min }}, \theta_{\text {max }}\right]$ hence $\theta \in\left(\theta_{\text {min }}, \theta_{\text {max }}\right)$. The parameter range for the displacement and the angle are be defined at the beginning of the ISAR process. The process will split the range into grids. Each grid will be evaluated by the process and the best one will be chosen.

The hypothetical location at time $t$ can be calculated by

$$
a_{t, j}^{\prime}=a_{t-1}+v_{t, j}
$$

The known location of $m$-th reference tag can be expressed as

$$
\boldsymbol{b}_{\boldsymbol{m}}=\left[x_{m}, y_{m}\right]
$$

The distance between the hypothetical position of the antenna and the known location of the reference tag at time $t$ is

$$
d_{t, j, m}=\left\|\boldsymbol{a}_{t, j}^{\prime}-\boldsymbol{b}_{\boldsymbol{m}}\right\|
$$

Where $\|\cdot\|$ is the norm operator of the distance vector.

At time $t$, the relative received phase which is measured by the reader from the $m$-th reference tag is

$$
\Delta \phi_{t, m}=\phi_{t, m}-\phi_{1, m}
$$

The relative received phase of $M$ reference tags can be expressed as

$$
\Delta \boldsymbol{\Phi}_{t}=\left[\Delta \phi_{t, 1}, \ldots, \Delta \phi_{t, m}, \ldots, \Delta \phi_{t, M}\right]^{T}
$$


For each hypothetical position of the moving antenna, the expected calculated phase can be calculated by

$$
\phi_{t, j, m}^{\prime}=\left(\phi_{0}+\frac{4 \pi d_{t, j, m}}{\lambda}\right) \bmod 2 \pi
$$

The relative expected phase can be written as

$$
\Delta \phi_{t, j, m}^{\prime}=\phi_{t, j, m}^{\prime}-\phi_{1, j, m}^{\prime}
$$

The sequence of relative expected phase can be written in vector form as

$$
\Delta \boldsymbol{\Phi}_{t, j}^{\prime}=\left[\Delta \phi_{t, j, 1}^{\prime}, \ldots, \Delta \phi_{t, j, m}^{\prime}, \ldots, \Delta \phi_{t, j, M}^{\prime}\right]^{T}
$$

The matching function defined as

$$
C_{t, j}=\exp \left(-j\left(\Delta \Phi_{t, j}^{\prime}-\Delta \Phi_{t}\right)\right)
$$

is a measure of the difference between the expected and measured phase for each hypothetical position of the moving antenna.

The probability for each hypothetical position of the moving antenna can be calculated as the sum of the result of the matching function

$$
\mathrm{P}_{t, j}=\left|\sum_{m=1}^{M} \mathbf{c}_{t, j}\right|
$$

The estimated position of the moving antenna at time $t$ would be the position with the largest probability

$$
\boldsymbol{a}_{\boldsymbol{t}}=\arg \max _{\boldsymbol{a}_{\boldsymbol{t}, j}^{\prime}} \mathrm{P}_{t, j}
$$

After obtaining the estimated position at time $t$, the process described above will repeat to estimate the position at time $t+1$. And finally, an estimated trajectory of the moving antenna can be express as

$$
A=\left[a_{1}, \ldots, a_{t}, \ldots, a_{T}\right]
$$

\section{ISAR-SAR}

After obtaining one estimated trajectory of the moving antenna, the location of the reference tags is estimated by applying the SAR algorithm to the recorded phases of the reference tags using the estimated trajectory. The error between the real location and estimated location of all reference tags provides an accuracy check. By changing the ISAR algorithm parameters; the range of the length of the displacement $L$ and the range of the angle of direction $\vartheta$, a new trajectory can be calculated. After applying SAR based on this new estimated trajectory, a different error in the know tag locations would be obtained. The localization error is compared with the expected localization error $E$. If it is larger than $E$, the previous trajectory would be abandoned, and a new trajectory will be estimated with different parameters. This process corresponds to the loop part as described in the flowchart as shown in Fig. 3.2. If no result smaller than $E$ is found for a long-running time, the $E$ could be increased to get a trajectory. The final trajectory would be the best fitting trajectory based on which the localization error of reference tags reaches the smallest value. This best-fitting trajectory will be used to locate target tags using the SAR method described in Section III-A.

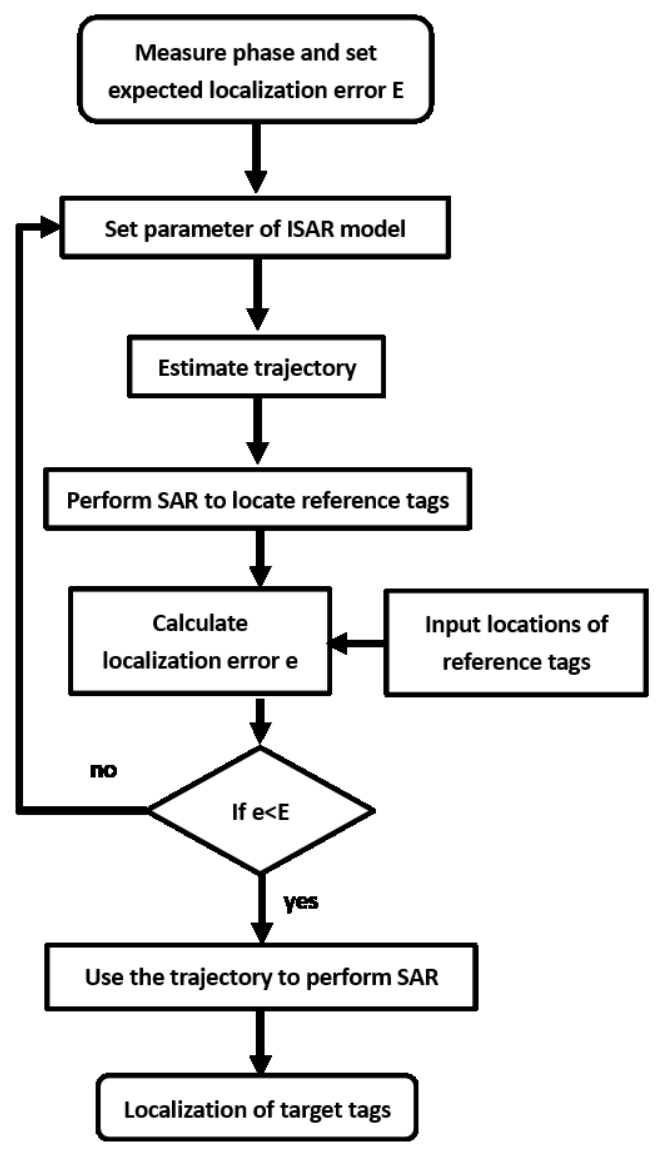

Fig. 3.2. The flow chart of ISAR-SAR method

\section{RESULTS WITH A STRAIGHT-LINE TRAJECTORY}

The first experiment was carried out in an indoor environment as shown in Fig. 4.1(b). As shown in Fig. 4.1(a), two rows of reference tags (black circles) and one row of target tags (blue crosses) were used. The antenna is moved along a straight-line trajectory which is shown by the blue line in Fig. 4.1(1). The LiDAR trajectory is estimated from fixed boards which are placed at the edges of the area at a known location. Using the shortest distance measured to each board, a 2D position estimate can be found. The trajectory is assumed to be the sequence of position estimates. The initial position of the robot is measured before tests and is at $(0.09,0.17)$. The first row of reference tags was placed $1.2 \mathrm{~m}$ away from the intended trajectory of the antenna while the second row of reference tags was placed $2 \mathrm{~m}$ away. In the middle of two rows of reference tags, there is one row of target tags. Both the distance between the rows and the distance between tags within rows is $0.4 \mathrm{~m}$. As shown in Fig. 4.1(a), the first tag of the first row was placed at $(0.2,1.2)$ and the first tag of the third row was placed at $(0.4$, $2.0)$ while the first tag of the target tags was at $(0.3,1.6)$. 


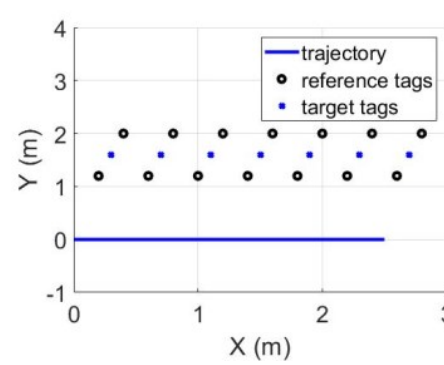

(a) Configuration of tags

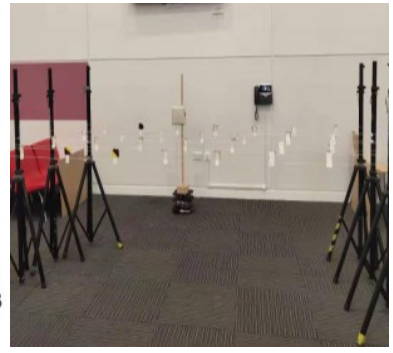

(b) Picture of the environment

Fig. 4.1. Experiment setup

Table I shows the results of the mean absolute error (MAE) of localization for reference tags during the ISAR process. When $\boldsymbol{l}_{\boldsymbol{m i n}} / \boldsymbol{l}_{\boldsymbol{m a x}}$ is varied, a new trajectory is estimated. As a result, the localization error of reference tags also varies. Table I shows that when the parameter is 3.5/5.0, the MAE of reference tags reaches a minimum $(17.49 \mathrm{~cm})$.

TABLE I. EFFECT OF PARAMETERS ON ISAR PROCESS

\begin{tabular}{|c|c|c||c|c|c||c|c|c|}
\hline \multicolumn{7}{|c|}{ The MAE of localization of reference tags $(\mathrm{cm})$} \\
\hline $\boldsymbol{l}_{\min }$ & $\boldsymbol{l}_{\max }$ & MAE & $\boldsymbol{l}_{\min }$ & $\boldsymbol{l}_{\max }$ & $\mathrm{MAE}$ & $\boldsymbol{l}_{\min }$ & $\boldsymbol{l}_{\max }$ & MAE \\
\hline \hline $\mathbf{1 . 5}$ & $\mathbf{5 . 0}$ & 45.17 & $\mathbf{1 . 5}$ & $\mathbf{5 . 5}$ & 26.07 & $\mathbf{1 . 5}$ & $\mathbf{6 . 0}$ & 38.22 \\
\hline $\mathbf{2 . 0}$ & $\mathbf{5 . 0}$ & 38.03 & $\mathbf{2 . 0}$ & $\mathbf{5 . 5}$ & 19.94 & $\mathbf{2 . 0}$ & $\mathbf{6 . 0}$ & 53.17 \\
\hline $\mathbf{2 . 5}$ & $\mathbf{5 . 0}$ & 27.36 & $\mathbf{2 . 5}$ & $\mathbf{5 . 5}$ & 24.56 & $\mathbf{2 . 5}$ & $\mathbf{6 . 0}$ & 71.23 \\
\hline $\mathbf{3 . 0}$ & $\mathbf{5 . 0}$ & 22.48 & $\mathbf{3 . 0}$ & $\mathbf{5 . 5}$ & 33.92 & $\mathbf{3 . 0}$ & $\mathbf{6 . 0}$ & 86.17 \\
\hline $\mathbf{3 . 5}$ & $\mathbf{5 . 0}$ & 17.49 & $\mathbf{3 . 5}$ & $\mathbf{5 . 5}$ & 48.04 & $\mathbf{3 . 5}$ & $\mathbf{6 . 0}$ & 97.49 \\
\hline $\mathbf{4 . 0}$ & $\mathbf{5 . 0}$ & 23.06 & $\mathbf{4 . 0}$ & $\mathbf{5 . 5}$ & 63.17 & $\mathbf{4 . 0}$ & $\mathbf{6 . 0}$ & 107.78 \\
\hline
\end{tabular}

Fig. 4.2 shows measured and ISAR calculated trajectories that are very close to each other. Fig. 4.3 is a zoomed-in view of Fig. 4.2 and shows more details of measured and estimated trajectories. The black lines represent the error vectors and show the corresponding relationship between two trajectories lining points corresponding to the same time step. The difference between the trajectories is of the order of a centimetre.

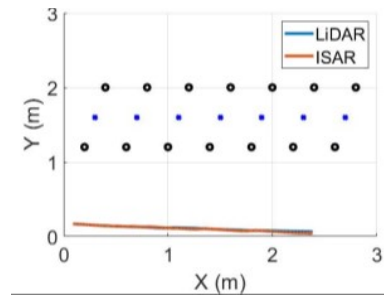

Fig. 4.2. Trajectory results of one of the experiments

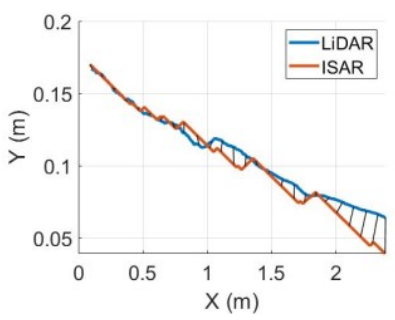

Fig. 4.3. Comparison of sample points between LiDAR trajectory and ISAR trajectory

Table II shows the error between the measured trajectory and estimated trajectory along the $\mathrm{x}$-axis and $\mathrm{y}$-axis of ten tests. The mean of MAE of ten tests along the $\mathrm{x}$-axis and $\mathrm{y}$-axis is around $3 \mathrm{~cm}$ and $2 \mathrm{~cm}$ respectively. As shown in Table II, except for Test 2, the MAE along the $\mathrm{x}$-axis of other tests are smaller than $5 \mathrm{~cm}$ and the MAE along the y-axis of ten tests as well as the

MAE of total error are within $5 \mathrm{~cm}$ which is similar to the datasheet accuracy of the LiDAR sensor. ( $\pm 15 \mathrm{~mm}$ within 500 $\mathrm{mm}$ and $\pm 5.0 \%$ when the distance is $500 \sim 3500 \mathrm{~mm}$ [32]), so the recorded differences are within the error bounds of the LiDAR itself.

It could be expected that the ISAR would provide a greater accuracy in the $\mathrm{x}$-direction due to the large aperature of the reference array in this direction. However, it is also likely that deviations from the straight-line track will be greatest in the $\mathrm{x}$ direction as well which would account for the larger MAE seen.

TABLE II. DIFFERENCE BETWEEN TRAJECTORY MEASURED BY LIDAR AND ESTIMATED BY ISAR

\begin{tabular}{|c||c|c|c|}
\hline \multicolumn{3}{|c|}{ MAE (cm) } & Total \\
\hline \hline Test & x-axis & y-axis & 5.83 \\
\hline 1 & 3.21 & 4.85 & 10.88 \\
\hline 2 & 10.67 & 2.90 & 3.33 \\
\hline 3 & 2.44 & 1.18 & 3.77 \\
\hline 4 & 3.00 & 0.81 & 4.63 \\
\hline 5 & 1.11 & 4.01 & 3.66 \\
\hline 6 & 3.56 & 1.36 & 3.03 \\
\hline 7 & 2.14 & 1.26 & 4.03 \\
\hline 8 & 1.14 & 3.67 & 3.08 \\
\hline 9 & 1.67 & 0.62 & 5.09 \\
\hline 10 & 4.60 & 0.93 & 4.73 \\
\hline \hline Mean & 3.35 & 2.16 & \\
\hline
\end{tabular}

After obtaining the estimated best-fitting trajectory, SAR is carried out to calculate the location of target tags. Both the trajectory measured by LiDAR and the trajectory estimated by ISAR are used to perform the SAR localization of target tags. The results are shown in Fig. 4.4

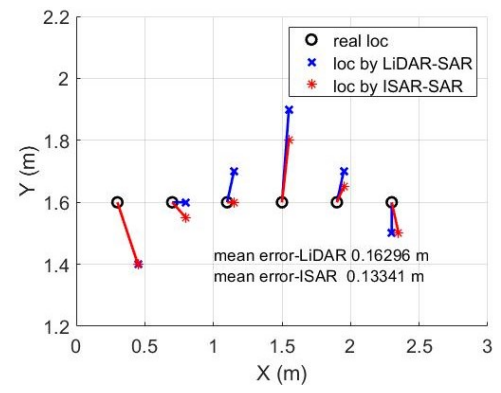

Fig. 4.4 Localization results of target tags of one of experiments

Black circles represent the actual location of target tags, blue crosses show the estimated location using the LiDAR trajectory while red stars show the estimated location using the bestfitting trajectory estimated by the ISAR method. It can be seen that two trajectories derived from different methods provide similar localization accuracy. The mean error of localization by using LiDAR trajectory is about $16 \mathrm{~cm}$ while that of the ISAR trajectory is around $13 \mathrm{~cm} .10$ tests have been carried out and mean localization error value as well as the mean localization error in percentage with respect to the minimum reader to tag range $(1.6 \mathrm{~m})$, have been summarized in Table III. The mean localization error of 10 tests by using LiDAR and ISAR methods is the same $(15 \mathrm{~cm})$. It is interesting to note that the error vectors are generally consistent between the LiDAR and 
ISAR, we believe this indicates that multipath propagation, which is the same in both cases, is one of the main sources of error. Many other factors, such as non-ideal phase offset [33], errors on locations of reference tags, configuration of reference tags, the error of the trajectory estimation, may also affect the accuracy of localization.

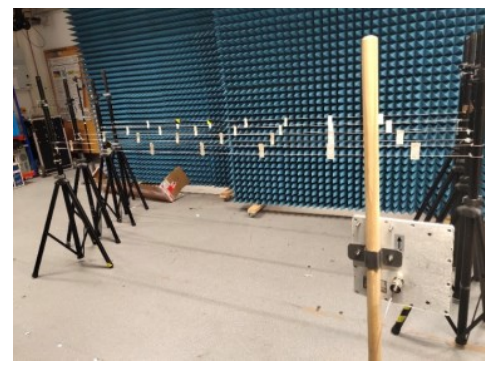

Fig. 4.5. Experiment setup

As shown in Fig.4.5, 10 tests have been carried out in a controlled environment with anechoic materials to reduce the largest reflections. The arrangement of the tags is as and intended antenna trajectory are both as shown in Fig.4.2 to allow the effect of the environment to be evaluated. The localization results are comparatively better than those achieved without the use of anechoic materials. The mean error of localization for target tags by LiDAR-SAR is $13.71 \mathrm{~cm}$ while the mean error by ISAR-SAR is $14.76 \mathrm{~cm}$. This might indicate that anechoic materials slightly reduce the multipath effect which results in higher accuracy. However, for localization error, the improvement is not significant compared with the results in Table III. Because anechoic materials only reduce reflections from surrounding objects and the ground reflection remains unaffected as previous tests, the ground reflection might be the main source of error for localization of target tags. For ISAR-SAR method, since the trajectory is estimated based on reference tags, the configuration of reference tags and the error of the trajectory estimation may also affect the accuracy of localization for target tags.

TABLE III. MEAN LOCALIZATION ERROR VALUE OF 10 TESTS BY SAR WITH A STRAIGHT LINE TRAJECTORIES

\begin{tabular}{|c||c||c||c||c|}
\hline \multicolumn{1}{|c||}{} & \multicolumn{1}{c||}{$\begin{array}{c}\text { Uncontrolled environment } \\
\text { (Fig. 4.1(b)) }\end{array}$} & $\begin{array}{c}\text { Semi-anechoic environment } \\
\text { (Fig. 4.5) }\end{array}$ \\
\hline \hline Test & LiDAR (cm) & ISAR (cm) & LiDAR (cm) & ISAR (cm) \\
\hline \hline 1 & 16.83 & 19.05 & 11.86 & 13.07 \\
\hline 2 & 14.21 & 13.24 & 13.37 & 12.11 \\
\hline 3 & 14.63 & 15.89 & 14.47 & 16.00 \\
\hline 4 & 15.69 & 15.02 & 14.40 & 15.26 \\
\hline 5 & 13.96 & 14.46 & 12.60 & 14.55 \\
\hline 6 & 16.57 & 21.41 & 11.86 & 14.82 \\
\hline 7 & 12.72 & 12.70 & 15.17 & 11.71 \\
\hline 8 & 13.39 & 11.42 & 15.17 & 15.70 \\
\hline 9 & 16.30 & 13.34 & 14.40 & 14.76 \\
\hline 10 & 16.57 & 14.59 & 13.78 & 19.64 \\
\hline \hline Mean & 15.09 & 15.11 & 13.71 & 14.76 \\
\hline
\end{tabular}

\section{RESULTS WITH AN L-SHAPE TRAJECTORY}

It can be seen in Fig. 4.4, that the error is mainly along the yaxis, perpendicular to the straight-line trajectory. When the trajectory is a straight line, SAR algorithm lacks depth perception perpendicular to the antenna track, as there will be little change in the observed phase difference. As a result, the localization error is mainly from the error along the y-axis. To address this problem, an L-shape trajectory can be used.

The experiment setup is shown in Fig. 5.1(a). Some boards with known locations are placed at the edges of the area as references for LiDAR to estimate the location of the robot and the initial position of the robot is measured which is at $(0.1$, 2.08). The L-shape trajectory shown by the black line consists of two-part. The first trajectory parallel to the $\mathrm{x}$-axis is $3 \mathrm{~m}$ followed by a $2 \mathrm{~m}$ long trajectory parallel to the y-axis. Target tags shown by the green crosses are placed in the middle of reference tags shown by the blue circles. Due to the fact that there are many objects such as equipment and metal objects in the lab, anechoic materials were used to partially reduce the influence of the objects in the lab.

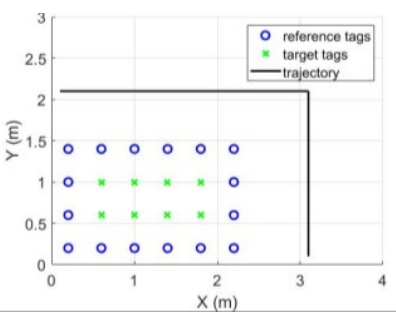

(a) Configuration of tags

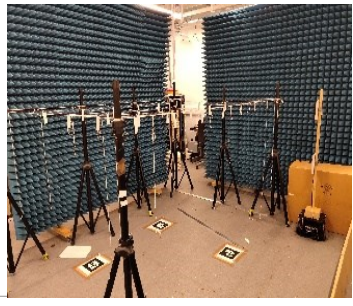

(b) Picture of the environment
Fig. 5.1. Experiment setup

The results of the trajectory estimation are shown in Fig. 5.2. The trajectory measured by LiDAR is shown by the blue line while the red line shows the trajectory estimated by the ISAR method. It can be seen that as the trajectory gets longer the error between the measured (LiDAR) and estimated (ISAR) trajectory also increases due to the cumulative error representing a random walk.

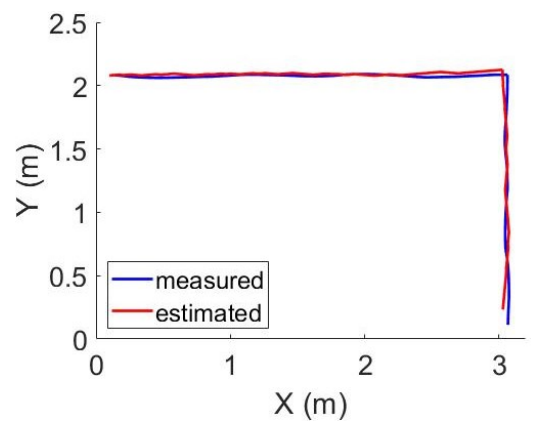

Fig. 5.2. Trajectory results of one of the experiments

Fig. 5.3 shows the error in a trajectory estimated by ISAR along the $\mathrm{x}$-direction and $\mathrm{y}$-directions for the portion of the track parallel to the $\mathrm{x}$ axis. The MAE along the $\mathrm{x}$-axis is 3.07 $\mathrm{cm}$ and the MAE along the $y$-axis is $1.43 \mathrm{~cm}$. Fig. 5.3(a) shows 
a clear trend that the error rises and reaches around $8 \mathrm{~cm}$ which is due to the cumulative error and will have a negative effect on the estimation for trajectory along the y-direction

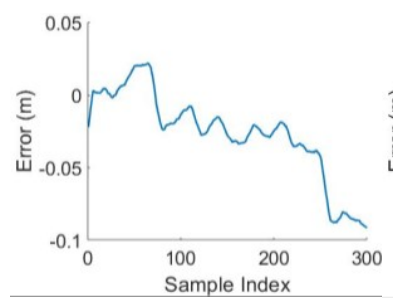

(a) Errors along $x$-axis

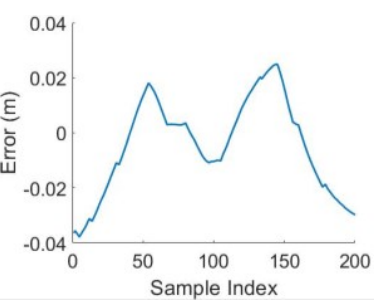

(b) Errors along y-axis
Fig. 5.3. Errors between ISAR trajectory and LiDAR trajectory along $\mathrm{X}$-direction

The error for the portion of the trajectory parallel to the $y$ axis is shown in Fig. 5.4. Fig. 5.4(a) shows the error in the $x-$ direction while Fig. 5.4(b) shows the error in the y-direction. The MAE along the $\mathrm{x}$-axis is $1.59 \mathrm{~cm}$ and the MAE along the $y$-axis is $7.77 \mathrm{~cm}$. As mentioned above, the large error along $\mathrm{y}-$ axis which could reach around $16 \mathrm{~cm}$ is partially due to the cumulative error. Another reason for larger trajectory error is that fewer reference tags locations are used along the $y-$ direction (four) compared with six along the $\mathrm{x}$-direction resulting in a smaller effective aperture.

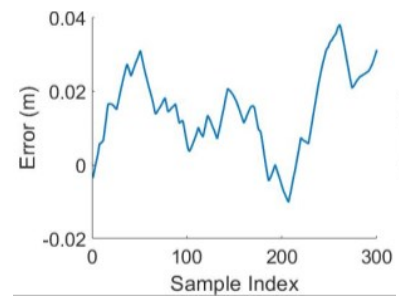

(a) Errors along $x$-axis

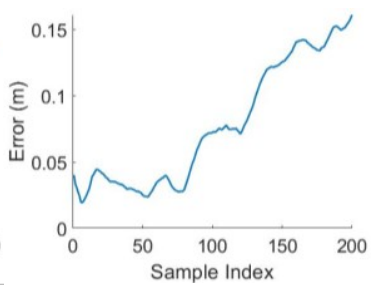

(b) Errors along y-axis
Fig. 5.4. Errors between ISAR trajectory and LiDAR trajectory along $\mathrm{y}$-direction

Five tests were carried out and the results of the trajectory estimation are summarized in Table IV.

TABLE IV. DIFFERENCE BETWEEN TRAJECTORY MEASURED BY LIDAR AND ESTIMATED BY ISAR

\begin{tabular}{|c||c|c|c||c|c|c|}
\hline \multicolumn{7}{|c|}{ MAE $(\mathrm{cm})$} \\
\hline & Trajectory along x-direction & \multicolumn{3}{l|}{ Trajectory along y-direction } \\
\hline \hline Test & x-axis & y-axis & Total & x-axis & y-axis & Total \\
\hline \hline 1 & 1.53 & 8.30 & 9.45 & 8.30 & 9.40 & 12.68 \\
\hline 2 & 8.00 & 6.23 & 8.01 & 0.68 & 7.50 & 10.36 \\
\hline 3 & 7.05 & 2.00 & 7.22 & 1.38 & 4.20 & 4.80 \\
\hline 4 & 3.07 & 1.43 & 3.63 & 1.59 & 7.77 & 8.03 \\
\hline 5 & 4.16 & 2.43 & 4.79 & 2.17 & 5.26 & 5.93 \\
\hline \hline Mean & 5.76 & 4.08 & 6.62 & 2.82 & 6.83 & 8.36 \\
\hline
\end{tabular}

Fig. 5.5 shows an example ambiguity function for localization of a tag by SAR in order to show the difference between the straight-line and L-shape trajectory. As mentioned in Section III, the L-shape trajectory could be divided into two straight-line trajectories separated by the indifferentiable turning point. Accordingly, two probability maps are calculated. Fig. 5.5(a) shows the probability map with a straight-line trajectory along $\mathrm{x}$-direction while Fig. 5.5(b) is the result when the trajectory is along the y-direction. As explained above, the ambiguity area which is shown by the yellow area in Fig 5.5(a) is extended along the y-axis while in Fig 5.5(b) it is more extended along the $x$-axis. If the trajectory is only a straight line as shown in Fig. 5.5(a), the lack of depth resolution along with influences from the environment such as multipath, results in a relatively large error in the y position as shown by the red cross.

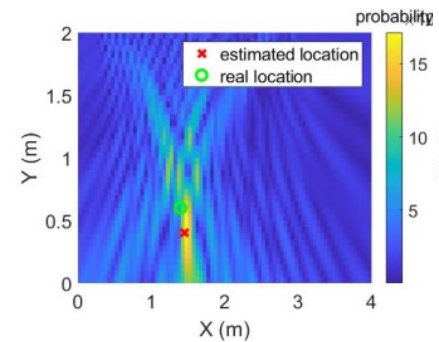

(a) Trajectory along $\mathrm{x}$-direction

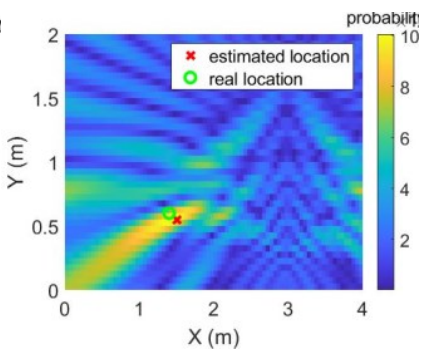

(b) Trajectory along y-direction
Fig. 5.5. Probability heatmap with a straight-line trajectory

Fig. 5.6 shows the result of localization using an L-shape. By combining both results along $\mathrm{x}$-direction and $\mathrm{y}$-direction (the product of 5.5(a) and 5.5(b)), the localization error is reduced as shown by the red cross which is much closer to the actual location shown by the green circle.

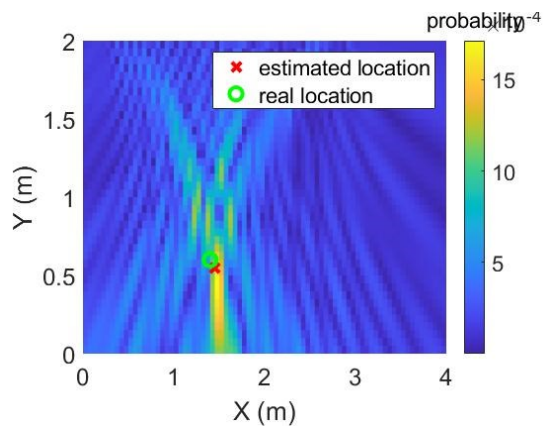

Fig. 5.6. Probability heatmap with an L-shape trajectory

Fig. 5.7 shows the result of localization for all target tags using an L-shape trajectory. Black circles are the real locations of target tags. Locations estimated by LiDAR-SAR are shown by green crosses while red crosses represent locations estimated by ISAR-SAR. The mean error for LiDAR-SAR is $7.95 \mathrm{~cm}$ and for ISAR-SAR is $4.38 \mathrm{~cm}$.

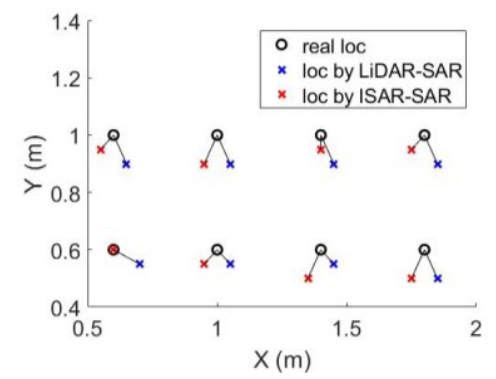

Fig. 5.7. Localization results of target tags of one of experiments 
The results of five tests are summarized in Table V. With an L-shape trajectory, LiDAR-SAR could reduce localization error to less than $10 \mathrm{~cm}$. In some tests, ISAR-SAR with an Lshape trajectory leads to a larger localization error than the straight trajectory. Since the straight-line trajectory is a subset of the L-shaped data, it can be concluded that in this case the $2^{\text {nd }}$ half of the L-shaped trajectory is contributing to increased error. This may be due to the different aperture of the reference tags in the y direction. As shown in Fig. 5.1(a), there are only four different locations of reference tags along the y-direction while there are six different locations of reference tags along the $\mathrm{x}$-direction this will reduce the accuracy of trajectory estimation along the y-direction and reduce the accuracy for localization using the L-shape trajectory. The initial location of the $2^{\text {nd }}$ half of the trajectory is also dependent on the error at the end of the $1^{\text {st }}$ trajectory. This also indicates that both number and configuration of reference tags have an influence on the accuracy of localization by the ISAR-SAR method. Sometimes ISAR-SAR could provide higher localization accuracy than LiDAR-SAR since the trajectory estimated by the ISAR-SAR method is based on reference tags. The process of estimating the best-fitting trajectory includes the effect of multipath so the best-fitting trajectory could mitigate the influence of multipath for localization by SAR.

TABLE V. MEAN LOCALIZATION ERROR VALUE OF 10 TESTS BY SAR WITH TWO TRAJECTORIES

\begin{tabular}{|c||c|c||c|c|}
\hline \multicolumn{5}{|c|}{ Localization error (cm) } \\
\hline \multicolumn{1}{|c||}{ Test } & $\begin{array}{c}\text { Straight } \\
\text { line }\end{array}$ & L shape & $\begin{array}{c}\text { Straight } \\
\text { line }\end{array}$ & L shape \\
\hline \hline 1 & 17.86 & 10.15 & 14.10 & 7.47 \\
\hline 2 & 11.91 & 7.95 & 1.25 & 4.38 \\
\hline 3 & 8.09 & 7.18 & 2.5 & 3.75 \\
\hline 4 & 8.61 & 7.58 & 6.03 & 5.77 \\
\hline 5 & 15.33 & 6.29 & 6.29 & 7.68 \\
\hline \hline Mean & 12.36 & 7.83 & 6.03 & 5.79 \\
\hline
\end{tabular}

\section{CONCLUSION}

This paper proposes a novel ISAR-SAR localization method with an ISAR based trajectory estimation algorithm by deploying reference tags. Low-cost passive RFID tags with known locations are used instead of additional sensors and infrastructures to estimate the trajectory of the mobile platform. An ISAR-SAR loop exploits the known location of reference tags to adjust the estimated trajectory in order to improve localization accuracy. Experimental results with a straight-line trajectory show that the MAE between the ISAR estimated and LIDAR measured trajectory is less than $5 \mathrm{~cm}$. When the estimated trajectory is used to find the location of target tags with unknown locations, the straight-line trajectory provides a $15 \mathrm{~cm}$ localization accuracy for targets tags; similar to the accuracy achieved using LiDAR-based antenna trajectory measurement. Experiments using an L-shape trajectory demonstrate more accurate localization. LiDAR-SAR with using only a straight-line segment of the trajectory could provide $12 \mathrm{~cm}$ accuracy (an improvement on earlier straight trajectory measurements owing to changes in the measurement environment), while the full L-shape trajectory could reach $8 \mathrm{~cm}$ accuracy. The proposed ISAR-SAR method performs much better still with a $6 \mathrm{~cm}$ accuracy.

\section{FUTURE WORK}

Since the ISAR-SAR method depends on reference tags, future work may focus on the effect of the number and configuration of reference tags and the influence of the location variance of reference tags. Moreover, since the ISAR-SAR method is designed for trajectory estimation and the fact that the performance of LiDAR sensor may degrade in a complicated environment, future research may also include combination of the ISAR-SAR method, which can be used as a complementary method, and multiple sensors from the robot such as the LiDAR sensor to further improve the performance of the system in both tracking of the moving platform and localization of targets.

\section{ACKNOWLEDGEMENT}

The authors would like to thank the Beijing Institute of Aerospace Control Devices (BIACD) for their support through the RFID system Optimization for smart manufacturing using Mobile rEader (ROME) project.

\section{REFERENCES}

[1] H. Ding et al., "Trio: Utilizing Tag Interference for Refined Localization of Passive RFID," 2018 IEEE INFOCOM 2018 - IEEE Conference on Computer Communications, Honolulu, HI, USA, pp. 828-836.

[2] Baha Aldin, N., Erçelebi, E. \& Aykaç, M. An Accurate Indoor RSSI Localization Algorithm Based on Active RFID System with Reference Tags. Wireless Pers Commun 97, 3811-3829 (2017).

[3] K. Nur, M. Morenza-Cinos, A. Carreras, and R. Pous, "Projection of RFID-Obtained Product Information on a Retail Stores Indoor Panoramas", IEEE Intelligent Systems, vol. 30, no. 6, pp. 30-37, Nov.-Dec. 2015.

[4] W. Lu, G. Q. Huang, and H. Li, "Scenarios for applying RFID technology in construction project management," in Automation in Construction, vol. 20, no. 2, pp. 101-106, 2011.

[5] R. Flanagan and W. Lu, "Making informed decisions in product-service systems," in IMechE Conference, Knowledge and Information Management Through-Life, Institute of Mechanical Engineers, London, 2008.

[6] A. Alexander, S. Phillips, and G. Shaw, "Retail Innovation and Shopping Practices: Consumers' Reactions to SelfService Retailing," in Environment and Planning A: Economy and Space, 40(9), 2204-2221, 2008.

[7] J. Koch, J. Wettach, E. Bloch, and K. Berns, "Indoor localisation of humans, objects, and mobile robots with RFID infrastructure," in 7th International Con-ference on Hybrid Intelligent Systems, pp. 271-276, September 2007.

[8] Dardari, D., Falletti, E., \& Luise, M. (2012). Satellites and Terrestrials Radio Positioning's Techniques. Cambridge: Elsevier AcademicsPress

[9] Bilodeau, JS., Bouzouane, A., Bouchard, B. et al. An experimental comparative study of RSSI-based positioning algorithms for passive RFID localization in smart environments. J Ambient Intell Human Comput 9, 13271343 (2018). 
[10] Li, C., Tanghe, E., Plets, D., Suanet, P., Hoebeke, J., Poorter, E., \& Joseph, W. (2019). RePos: Relative Position Estimation of UHF-RFID Tags for Item-level Localization. 2019 IEEE International Conference on RFID Technology and Applications (RFID-TA), 357-361.

[11] Bernardini, F., Motroni, Nepa, Buffi, Tripicchio, \& Unetti. (2019). Particle Swarm Optimization in Multi-Antenna SAR-based Localization for UHF-RFID Tags. 2019 IEEE International Conference on RFID Technology and Applications (RFID-TA), 291-296.

[12] A. Motroni, P. Nepa, V. Magnago, A. Buffi, B. Tellini, D. Fontanelli, and D. Macii, "SAR-Based Indoor Localization of UHF-RFID Tags via Mobile Robot", 2018 International Conference on Indoor Positioning and Indoor Navigation (IPIN), Nantes, 2018, pp. 1-8.

[13] A. Motroni, P. Nepa, A. Buffi, P. Tripicchio, and M. Unetti, "RFID Tag Localization with UGV in Retail Applications", $20183^{\text {rd }}$ International Conference on Smart and Sustainable Technologies (SpliTech), Split, 2018, pp. 1-5.

[14] A. Buffi, M. R. Pino, and P. Nepa, "Experimental Validation of a SAR Based RFID Localization Technique Exploiting an Automated Handling System", IEEE Antennas and Wireless Propagation Letters, vol. 16, pp. 2795-2798, 2017.

[15] R. Miesen, F. Kirsch, and M. Vossiek, "UHF RFID localization based on synthetic apertures", IEEE Transactions on Automation Science and Engineering, vol. 10, no. 3, pp. 807-815, Jul. 2013.

[16] A. Buffi, A. Motroni, P. Nepa, B. Tellini, and R. Cioni, "A SAR-Based Measurement Method for Passive-Tag Positioning With a Flying UHFRFID Reader", IEEE Transactions on Instrumentation and Measurement, vol. 68, no. 3, pp. 845-853, March 2019.

[17] A. Buffi and B. Tellini, "Measuring UHF-RFID Tag Position via Unmanned Aerial Vehicle in Outdoor Scenario", 2018 IEEE $4^{\text {th }}$ International Forum on Research and Technology for Society and Industry (RTSI), Palermo, 2018, pp. 1-6.

[18] L. Qiu, Z. Huang, N. Wirström and T. Voigt, "3DinSAR: Object 3D localization for indoor RFID applications", 2016 IEEE International Conference on RFID (RFID), Orlando, FL, 2016, pp. 1-8.

[19] Y. Zhang, L. Xie, Y. Bu, Y. Wang, J. Wu, and S. Lu, "3Dimensional Localization via RFID Tag Array", 2017 IEEE 14th International Conference on Mobile Ad Hoc and Sensor Systems (MASS), Orlando, FL, 2017, pp. 353361 .

[20] A. Ascher, J. Lechner, S. Nosovic, P. Eschlwech, and E. Biebl, "3D localization of passive UHF RFID transponders using direction of arrival and distance estimation techniques", 2017 IEEE 2nd Advanced Information Technology, Electronic and Automation Control Conference (IAEAC), Chongqing, 2017, pp. 1373-1379.

[21] G. Alvarez-Narciandi, J. Laviada, M. R. Pino, and F. LasHeras, "3D location system based on attitude estimation with RFID technology", 2017 IEEE International Conference on RFID Technology \& Application (RFIDTA), Warsaw, 2017, pp. 80-82.

[22] A. Buffi, P. Nepa, and R. Cioni, "SARFID on drone: Drone-based UHF-RFID tag localization," in 2017 IEEE International Conference on RFID Technology \& Application (RFID-TA), 2017.
[23] Tzitzis, A., Megalou, S., Siachalou, S., Tsardoulias, E., Yioultsis, T., \& Dimitriou, A. (2019). 3D Localization of RFID Tags with a Single Antenna by a Moving Robot and "Phase ReLock". 2019 IEEE International Conference on RFID Technology and Applications (RFID-TA), 273278.Phase ReLock - Localization of RFID Tags by a Moving Robot

[24] Tzitzis, A., Megalou, S., Siachalou, S., Yioultsis, T., Kehagias, A., Tsardoulias, E., Dimitriou, A. (2019). Phase ReLock - Localization of RFID Tags by a Moving Robot. 2019 13th European Conference on Antennas and Propagation (EuCAP), 1-5.

[25] Siachalou, S., Megalou, S., Tzitzis, A., Tsardoulias, E., Bletsas, A., Sahalos, J., Dimitriou, A. (2019). Robotic Inventorying and Localization of RFID Tags, Exploiting Phase-Fingerprinting. 2019 IEEE International Conference on RFID Technology and Applications (RFIDTA), 362-367.

[26] E. DiGiampaolo and F. Martinelli, "A Passive UHF-RFID System for the Localization of an Indoor Autonomous Vehicle," in IEEE Transactions on Industrial Electronics, vol. 59, no. 10, pp. 3961-3970, Oct. 2012.

[27] E. DiGiampaolo and F. Martinelli, "Mobile Robot Localization Using the Phase of Passive UHF RFID Signals," in IEEE Transactions on Industrial Electronics, vol. 61, no. 1, pp. 365-376, Jan. 2014.

[28] Z. Liu, Z. Fu, T. Li, I. White, R. Penty and M. Crisp, "An ISAR-SAR based Localization Method using Passive UHF RFID System with Mobile Robotic Platform," 2020 IEEE International Conference on RFID (RFID), Orlando, FL, USA, 2020, pp. 1-7.

[29] Impinj, Inc, “ IMPINJ SPEEDWAY READER OVERVIEW, SOFTWARE TOOLS, ACCESSORIES, AND SPECIFICATIONS", Impinj”, Impinj Speedway R420 datasheet https://support.impinj.com/hc/enus/articles/202755388-Speedway-Reader-FamilyProduct-Brief-Datasheet

[30] ROBOTIS, INC., "Hardware Specifications", Turtlebot3 Waffle $\mathrm{Pi}$ datasheet http://emanual.robotis.com/docs/en/platform/turtlebot3/sp ecifications/

[31] Raspberry Pi Foundation, "Raspberry Pi Compute Module 3+ Raspberry Pi Compute Module 3+ Lite", Raspberry Pi datasheet https://www.raspberrypi.org/documentation/hardware/co mputemodule/datasheets/rpi_DATA_CM3plus 1p0.pdf

[32] ROBOTIS, INC, "360 Laser Distance Sensor LDS-01 (LIDAR)", [903-0258-000] 360 Laser Distance Sensor LDS-01 http://www.robotis.us/360-laser-distance-sensorlds-01-lidar

[33] Y. Ma, H. Liu, Y. Zhang and Y. Jiang, "The Influence of the Nonideal Phase Offset on SAR-Based Localization in Passive UHF RFID," in IEEE Transactions on Antennas and Propagation, vol. 68, no. 8, pp. 6346-6354, Aug. 2020, doi: 10.1109/TAP.2020.2982448. 


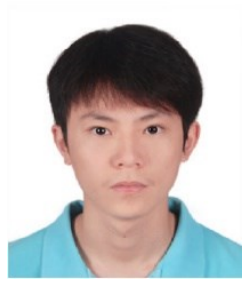

Zheng Liu received the B.Eng. degree from the University of Sheffield, Sheffield, U.K. in 2017 and the M.Res. degree in Engineering from the University of Cambridge in 2018. He is currently a Ph.D student within the CPS group. His current research focuses on passive RFID localisation using mobile platforms.

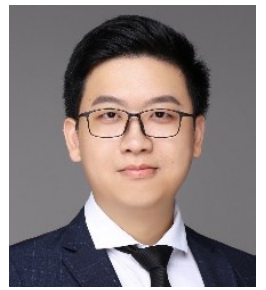

Zhe $\mathrm{Fu}$ received the B.Eng. degree in electronic information science and technology from the University of Shanghai for Science and Technology, China, in 2012, the M.Sc. degree in digital communications from the University of Bath in 2013, the M.Res. degree in photonics systems development from University College London in 2014, and the Ph.D. degree from the University of Cambridge, U.K., in 2018, where he is currently a Research Associate with the Centre for Photonic Systems. His research interests include RFID, IoT and robotic system design, RF circuits design, and algorithms for next generation cyber-physical systems.

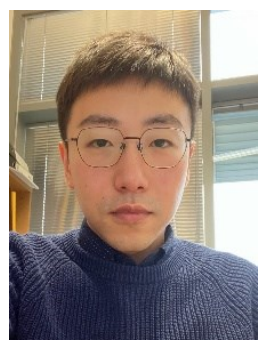

Tongyun Li (Member, IEEE) received the B.Eng. degree in electronics and computer engineering from the University of Aberdeen, in 2007, and the $\mathrm{Ph} . \mathrm{D}$. degree in engineering from the University of Cambridge, in 2012. He is currently a Research Associate with the Centre for Photonic Systems, Engineering Department, University of Cambridge. His research interests include digital radio over fiber, in-building wireless systems, next-generation wireless fronthaul systems, radio frequency identification (RFID), and photonic systems and subsystems.

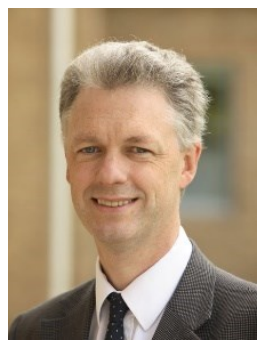

Ian H. White (Fellow, IEEE) received the B.A. and Ph.D. degrees from the University of Cambridge in 1980 and 1984, respectively. He was then appointed as a Research Fellow and an Assistant Lecturer with the University of Cambridge before becoming a Professor of physics with the University of Bath in 1990. In 1996, he joined the University of Bristol as a Professor of optical communications and became the Head of the Department of Electrical and Electronic Engineering in 1998, before returning to the University of Cambridge in 2001. In 2005, he became the Head of the School of Technology and subsequently the Chair, leaving the School of Technology to take up the position of a Pro-ViceChancellor for Institutional Affairs in
2010. He is a Co-Founder of Zinwave Ltd., and PervasID Ltd. $\mathrm{He}$ is currently the Vice-Chancellor of the University of Bath. He was a recipient of the Aron Kressel Award from the Institute of Electrical and Electronics Engineers in 2011. He is the Editor-in-Chief of Electronics Letters, and has published in excess of 250 journal papers. He is a Fellow of the Royal Academy of Engineering, Institution of Electrical Engineers, and the Institute of Electrical and Electronics Engineers.

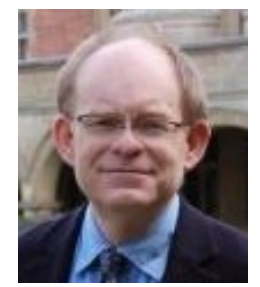

Richard V. Penty (Senior Member, IEEE) received the Ph.D. degree in engineering from the University of Cambridge, Cambridge, U.K., in 1989, for his research on optical fiber devices for signal processing applications, where he was a Science and Engineering Research Council Information Technology Fellow researching on all optical nonlinearities in waveguide devices. He is currently a Professor of photonics with the University of Cambridge, having previously held academic posts with the University of Bath and the University of Bristol. He has authored over 750 refereed journal and conference papers. His research interests include high speed optical communications systems, photonic integration, optical switching, and sensing systems. $\mathrm{He}$ is the Editor-in-Chief of the IET Optoelectronics Journal. He is a Fellow of the Royal Academy of Engineering and IET.

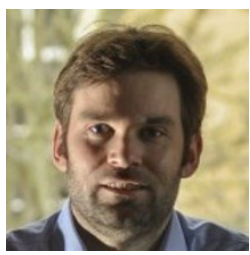

Michael Crisp (Member, IEEE) received the undergraduation and Ph.D. degrees in optical and RF systems, exploiting the benefits of optical fibres for the analog distribution of radio signals within buildings from the University of Cambridge, Cambridge, U.K., where he is currently a Lecturer in photonics and RF systems with the Engineering Department. He co-founded a spin out company PervasID. His current research interests include RF systems for next generation battery-less sensors and analog applications of photonic systems. He has remained in University of Cambridge researching on radio frequency identification systems, for which he was a recipient of the Royal Academy of Engineering Young Entrepreneurs Award in 2011 . 